\title{
Analysis of rosette modes of oscillations in rotating stars
}

\author{
Masao Takata ${ }^{1}$ and Hideyuki Saio ${ }^{2}$ \\ ${ }^{1}$ Department of Astronomy, School of Science, The University of Tokyo, \\ 7-3-1 Hongo, Bunkyo-ku, Tokyo 113-0033, Japan \\ email: takata@astron.s.u-tokyo.ac.jp \\ ${ }^{2}$ Astronomical Institute, Graduate School of Science, Tohoku University, \\ 6-3 Aramaki, Aoba-ku, Sendai 980-8578, Japan \\ email: saio@astr.tohoku.ac.jp
}

\begin{abstract}
We analyse the rosette modes of oscillations in rotating stars, which are characterised by rosette patterns of the kinetic-energy distribution on the meridional plane. Following our previous argument that these modes are generated by the rotation-induced interaction among eigenmodes with almost the same frequency, we discuss the structure of the rosette patterns base on the JWKB analysis. We also demonstrate that there exist nonaxisymmetric rosette modes.
\end{abstract}

Keywords. stars: interiors, stars: oscillations, stars: rotation

\section{Introduction}

The rosette modes constitute a new class of eigenmodes of oscillations in rotating stars. They have been discovered by Ballot et al. (2012), who numerically computed adiabatic eigenmodes of a polytropic model with index 3 that rotates uniformly at a few tens of percent of the break-up rate. Although the frequency of the modes is found in the range of that of gravity modes, it is not too low to be in the inertial domain, where the amplitude of eigenmodes is confined in the equatorial region. The most remarkable property of the modes is the fact that their kinetic-energy density distributes along rosette patterns on the meridional plane. Takata \& Saio (2013) have identified the physical origin of the rosette modes as close degeneracy in the frequency among eigenmodes that have successive values of the spherical degree with the same parity (in the absence of rotation). Those modes strongly interact with each other due to the Coriolis force to form a family of rosette modes. This process can be precisely described by quasi-degenerate perturbation theory. As further analysis along the same lines, this paper concentrates on the JWKB analysis and nonaxisymmetric rosette modes.

\section{Analysis and discussions}

An asymptotic analysis can be developed to explain the structure of the rosette patterns in a simple way. After deriving expressions of eigenfrequencies and eigenfunctions of gravity modes for a large spherical degree, we substitute those expressions into the formula of the quasi-degenerate perturbation theory. Assuming that eigenmodes are completely degenerate in the absence of rotation, and that the number of degenerate modes is infinite, we obtain simple expressions of the rosette patterns, which are depicted in Fig. 1. It is found that the rosette pattern of each mode is composed of eight curves (in general), which are specified by two parameters. The first parameter, $K$, indicates a common non-negative integer to all rosette modes that come from a given family of 


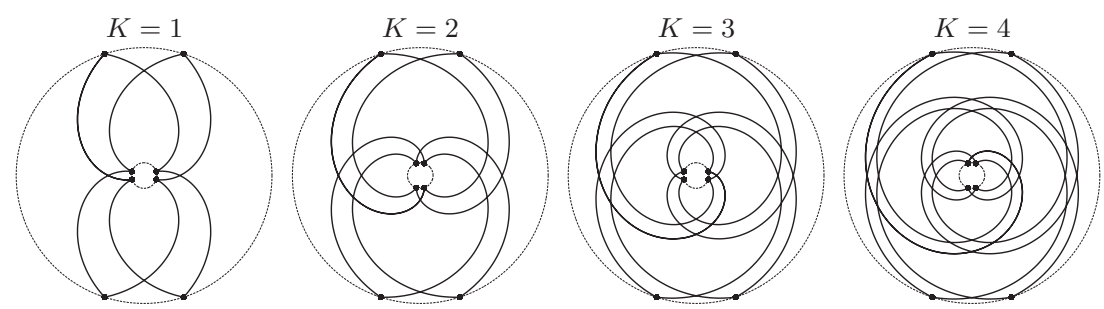

Figure 1. Schematic asymptotic structure of rosette modes on the meridional plane. The parameter $q$ is chosen to be $q=0.2$, whereas the parameter $K$ is given above each panel. Refer to the main text for the meanings of the parameters. The dashed circles represent the inner and outer turning points.
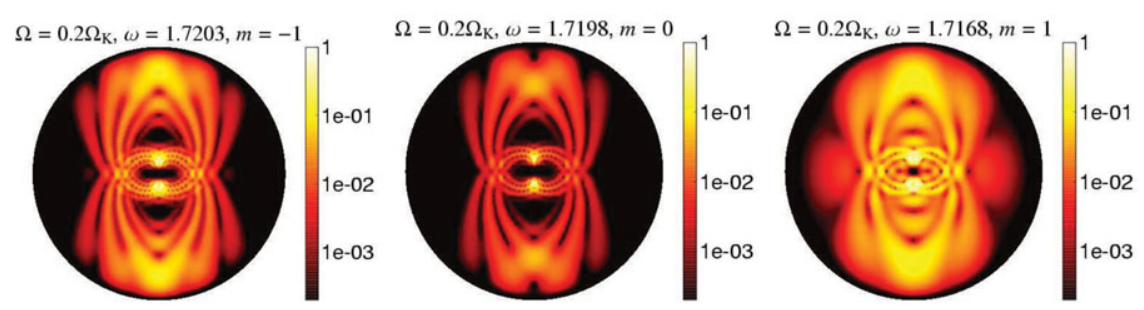

Figure 2. Distribution of the kinetic-energy density (multiplied by the square of the radius) of rosette modes on the meridional plane. The rotation rate, $\Omega$ (in units of the break-up rate, $\Omega_{\mathrm{K}}$ ), the dimensionless frequency, $\omega$, and the azimuthal order, $m$, are given above each panel.

close degeneracy. The larger is $K$, the lower is the frequency of the corresponding family. The difference in the polar angle between the two end points of each constituent curve is equal to $K \pi / 2$. The second parameter, $q$, is a fractional number between 0 and 1 . The tilt angle of each rosette pattern from the rotation axis is equal to $q \pi / 2$.

In the analysis of nonaxisymmetric rosette modes based on the quasi-degenerate perturbation theory, it turns out to be essential to assume that the Ledoux constant, $C_{n, l}$, is a small quantity on the same order as the rotation rate, $\Omega$. This assumption has been checked not only numerically, but also asymptotically for large values of the spherical degree. Because of this assumption, the leading-order perturbation to the frequency (in the corotating frame), $-m C_{n, l} \Omega$, where $m$ is the azimuthal order, is regarded as a second-order quantity. The rest of the analysis is essentially the same as in the axisymmetric cases. Examples of the thus-calculated nonaxisymmetric rosette modes are shown in Fig. 2. It is observed that the retrograde mode $(m=-1)$ shows a clearer rosette pattern than the prograde mode $(m=1)$.

\section{Conclusion and prospects}

The rosette modes have revealed a new aspect of the effect of rotaion on oscillations. Even more analysis is awaited to discuss topics such as the possibility of their excitation and their influence on angular-momentum transport in stars.

\section{References}

Ballot, J., Lignières, F., Prat, V., Reese, D. R., \& Rieutord, M. 2012, ASP-CS, 462, 389

Takata, M. \& Saio, H. 2013, PASJ, 65, 68 